\title{
Representation of a Distributed Database System for the Medical Purposes Using Oracle
}

Basam A. Mustafa
Ahmed A. Al-Saman

\section{College of Computer Science and Mathematics \\ University of Mosul, Mosul, Iraq}

Received on: 17/11/2011

Accepted on: 15/02/2012

\begin{abstract}
This research concerned with the designing and implementation of a distributed database system for the medical purposes. The system has been applied on dental clinic unit and statistics department at Al-Khansaa Educational Hospital at Mosul, and dental clinic unit at Woman Health Care Center at Mosul as a case study.

Client/server model has been used to implement the proposed system's architecture. The computers have been connected together through a local area network (LAN). Horizontal fragmentation technique has been used to distribute the database which achieved a good level of local autonomy. Oracle software were used and utilized to implement the system. It played a dramatic role in protecting data using combination of passwords and user roles hierarchies in addition to achieving transparency and data integrity concepts in the system. The proposed system simplified saving and retrieving data of dental clinics. It also provides dental clinics units and statistics department with necessary reports and statistics. The proposed system has the ability to automatically perform daily backup for the database in addition to the manual options for database backup and recovery.
\end{abstract}

Keywords: distributed database system, medical purposes, Oracle.

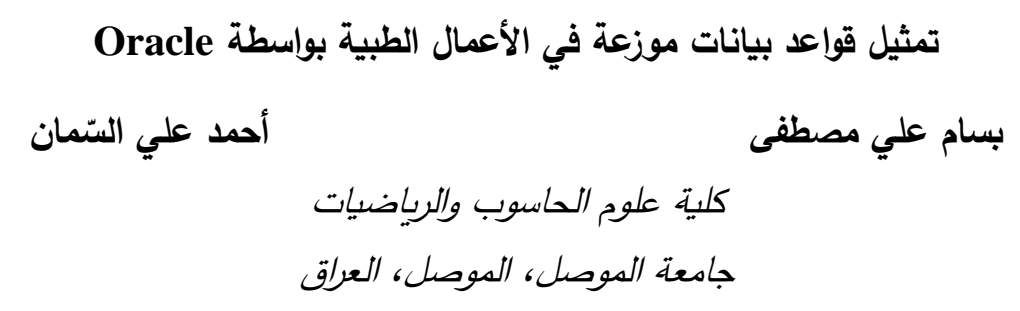

تاريخ قبول البحث: 2012/02/15

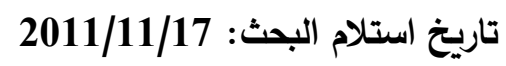


النظام وقد لعبت دوراً كبيراً في تحقيق الحماية للبيانات باستخدام كلمات المرور وهرميات من صلاحيات

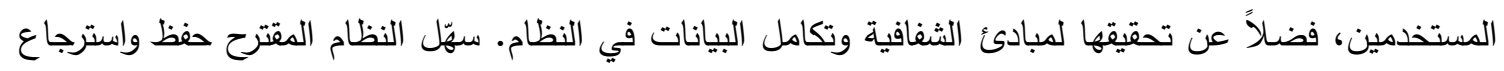

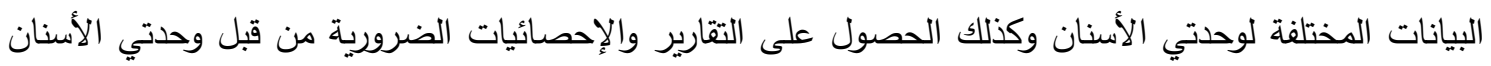
وشعبة الإحصاء. ويقوم النظام المقترح بالخزن الاحتياطي اليومي الأوتوماتيكي لقاعدة البيانات فضلاً عن توفير خياري الخزن الاحتياطي والاسترداد اليدويين.

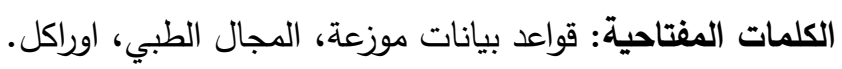

\section{Introduction}

- 1- 1 المقدمة

تعد أنظمة المعالجة الموزعة شكلا حديثا ومتطورا من أشكال المعالجة اللامركزية. ولقد ازداد الطلب

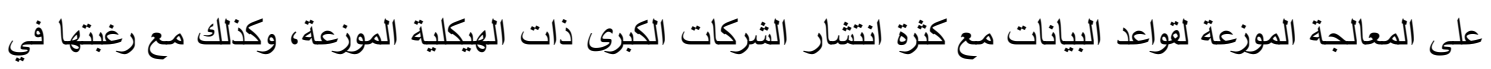
توزيع حمل المعالجة وحاجتها إلى أمن المعلومات وإجراء العمليات بأسرع وقت ممكن. ويمكن القول أن تكنولوجيا معالجة قواعد البيانات الموزعة هي نتاج التطور الحاصل في تقانات الاتصالات وتقانات قواعد البيانات [6].

\section{Database}

2- 2 - 2قاعدة البيانات

عبارة عن نظام حاسوبي لخزن السجلات هدفه الأساسي حفظ المعلومات والسماح للمستخدمين باسترجاع وتحديث البيانات بصورة أنموذجية وبدون تكرار لتخدم احتياجات المستخدمين ضمن المؤسسة، وتدار عن طريق

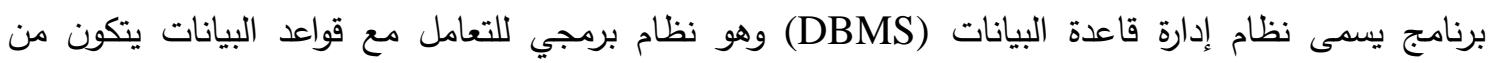

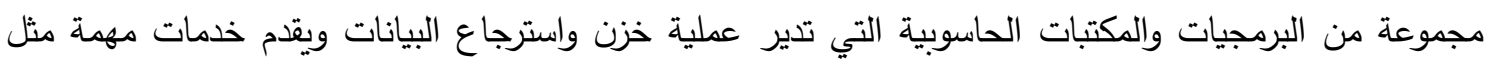
إدارة العمليات، السيطرة على الوصول المتزامن إلى البيانات، تكامل البيانات، وحماية البيانات. ويعتبر حلقة الوصل ولتئل بين المستخدمين وقاعدة البيانات [19]. والثكل (1) [24] يمثل موقع نظام إدارة قاعدة البيانات. و واجهة المسنخدم

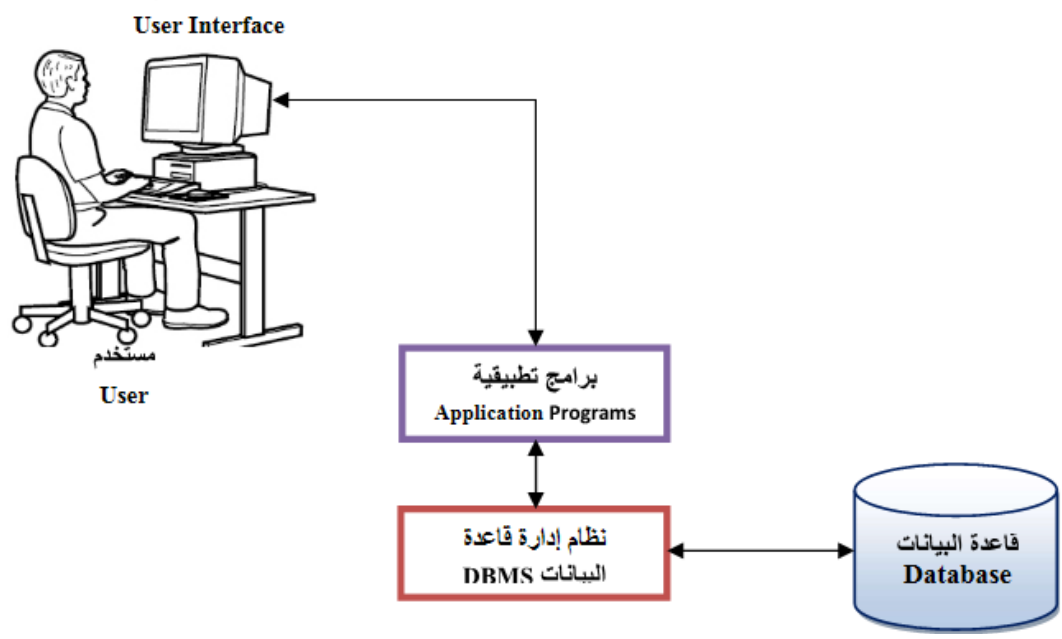

الشكل (1) موقع نظام إدارة قاعدة البيانات

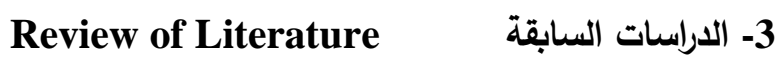

في عام 1982 قدم الباحثان Goodman N. و ورقةً بحثيةً أثارا فيها إلى مفاهيم السيطرة على الوصول المتزامن (concurrency control)، وتتاولا كذلك طرائق (خوارزميات) عديدة سابقة للسيطرة على ولى 
الوصول المتزامن في عمليات (القراءة-الكتابة) و عمليات (الكتابة-الكتابة) بالإضافة إلى اقتراح طرائق جديدة. وتم

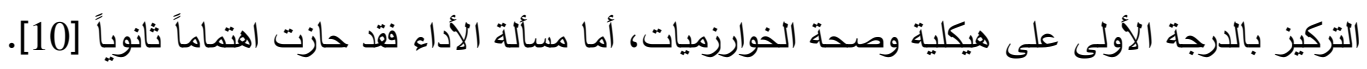
في عام 1997 قدم الباحثان Chakarvarthy S. Malinowski E. بحثاً تتاولا فيه المسائل المتعلقة بالتجزئة عند تصميم قواعد البيانات شيئية التوجه (object-oriented databases) وخاصةً التجزئة العمودية للكائنات، تعميماً للأعمال السابقة على قواعد البيانات العلائقية [16].

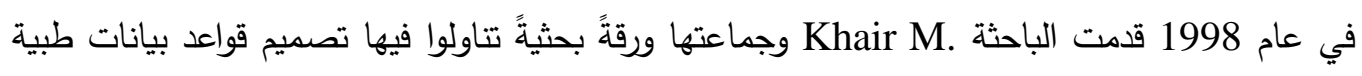

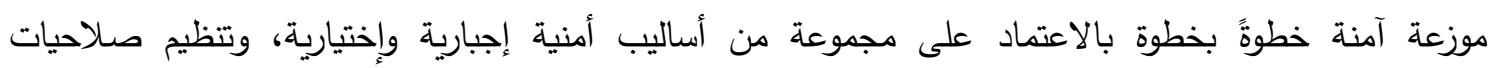

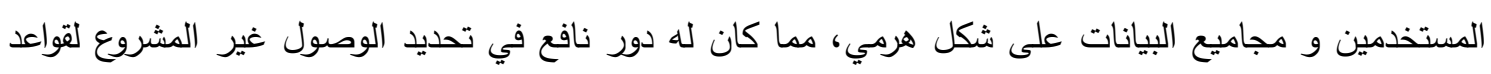
البيانات الطبية [14]. في عام 2002 قدم الباحثان .Labban R. S. و بحثاً تتاول شرحاً لإدارة المناقلات في

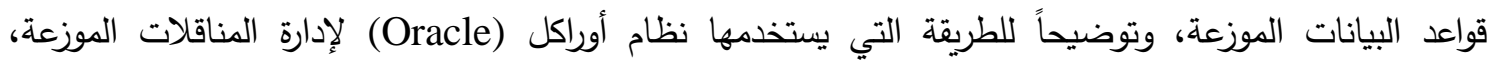

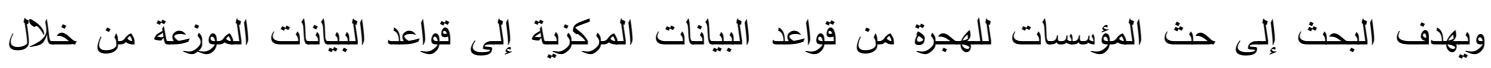
استخدام نظام إدارة قواعد بيانات أوراكل [7]. في عام 2004 قدمت الباحثة ظفر فخري الليلة رسالة ماجستير تضمنت استخدام إحدى تنظيمات قواعد البيانات الموزعة، وتطبيق هذا التتظيم على تصميم نظام الأفراد، ويتميز هذا التتظيم بقواعد بيانات مركزية مشتركة فئي

ووصول متعدد، وتم تقسيم العمل مابين هذه المواقع ضمن نظام للصلاحيات محدد من قبل مدير النظام [1]. في عام 2005 قدم الباحث مababaah H. بحثاً تضمن جزئين، حيث تتاول الجزء الأول أساسيات

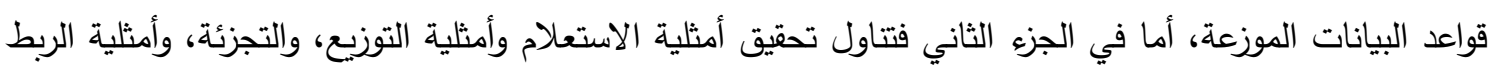
(join optimization)

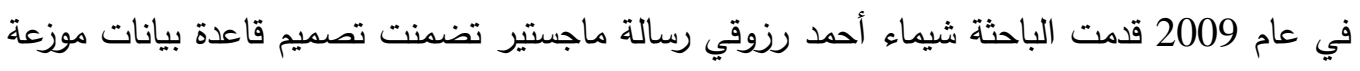

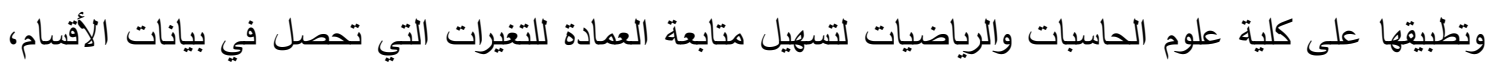

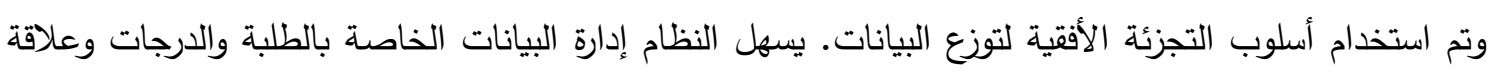

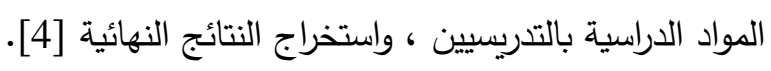

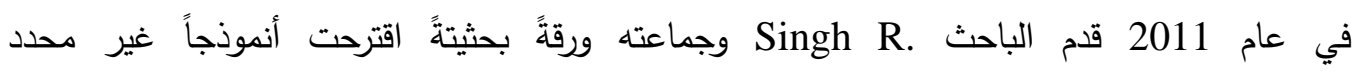
(stochastic model) مقترحة لتحقيق الأمثلية لخطة الوصول (access strategy) للاستفسارات الموزعة على المواقع في الشبكة، وكذلك قاموا بتحليل تأثيرات تغيير المعلمات الجينية (genetic parameters) على جودة تحقيق الأمثلية [20].

\section{Computer Networks}

4- 1 شبكات الحاسوب

تعرف شبكة الحاسوب بأنها مجموعة من الحواسيب المرتبطة مع بعضها بواسطة روابط الاتصال لتبادل

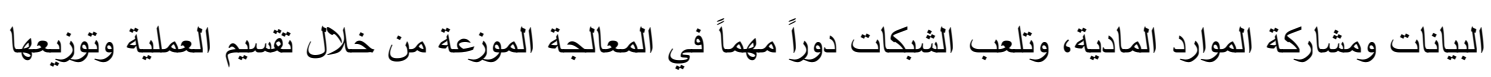
على الحاسوب المربوطة بالثبكة. والثكل (2) يوضح مكونات نظام اتصال البيانات [8][11]. 


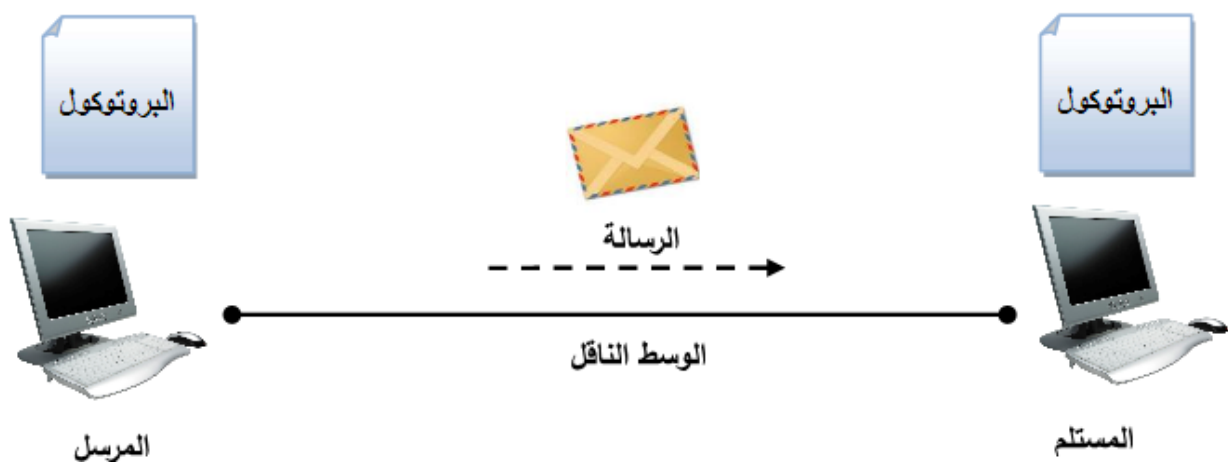

الشكل (2) مكونات نظام اتصال البيانات [11]

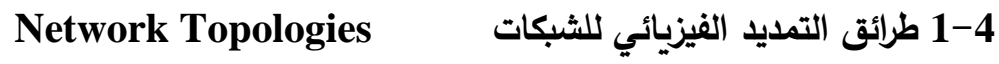

يعرف التمديد الفيزيائي للشبكة بأنه الطريقة الهندسية أو المعمارية التي يتم بها ربط أو تمديد الثبكة

فيزيائياً بمكوناتها المادية من روابط اتصال وأجهزة، وتوجد أربع طرائق أساسية للربط وهي الثبكة المعثّقة لثئة (mesh)، النجمة (star)، الخط الناقل (bus)، الحلقة (ring)، والهجينة (hybrid)، ولقد استخدمت الطريقة النجمية (star topology) لربط الثبكة فيزيائياً في النظام المقترح [8][13] (13) •

Classification of Network 4-2-4 تصنيف الثبكات

تصنّف الثبكات قياساً إلى حجمها إلى صنفين أساسيين هما الثبكات المحلية (Local Area Networks LAN)

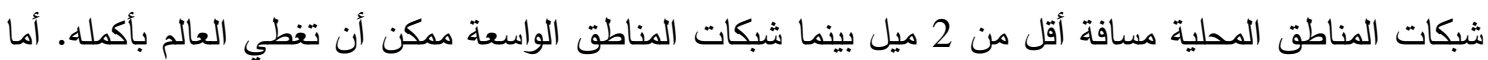
شبكات المناطق المدنية (Metropolitan Area Networks MAN) فهي أكبر حجماً من شبكات المناطق المحلية وتستخدم على مستوى المدينة وتغطي عشرات الأميال [11][1].

Transmission Media 3-4 وهي الأوساط المستخدمة في نقل المعلومات (الإشارات) من المرسل إلى المستلم في شبكات الحاسوب،

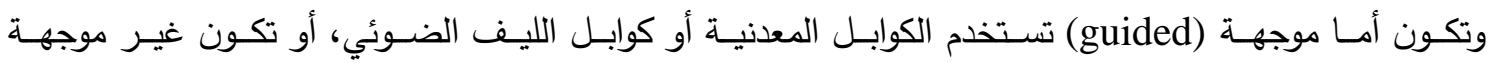
(unguided)

Networking Standards 4-4 المعايير القياسية للاتصالات في الشبكات من أجل الاتصال بين الحواسيب في الثبكات لابد من توفر معايير قياسية تتحكم بعملية الاتصال، فلهذا السبب تم إنثاء نماذج قياسية لتضم تلك المعايير ومنها أنموذج الاتصالات مابين الأنظمة المفتوحة • (TCP/IP Protocol Suite) TCP/IP و طاقم بروتوكولات (Open Systems Interconnection OSI) تضم تلك النماذج مجموعة من وظائف الاتصال مقسمة على شكل طبقات مختلفة يمكنها العمل بثكلٍ مستقل عن بعضها البعض، مما يساعد في التعديل على طبقة معينة من دون التعديل على الأنموذج ككل. تستخدم كل طبقة

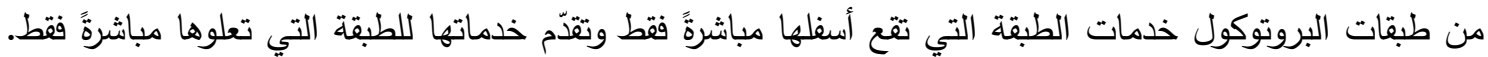
يمكن تطبيق مجموعة البروتوكولات ضمن البرمجيّات، التجهيزات أو ضمن خليطٍ من كليهما [13][8]. وتم استخدام بروتوكول TCP في النظام المقترح. 


\section{Relational Databases}

وهي عبارة عن قواعد بيانات تعتمد على الأنموذج العلائقي الذي وضع أساسه العالم Edgar F. Codd،

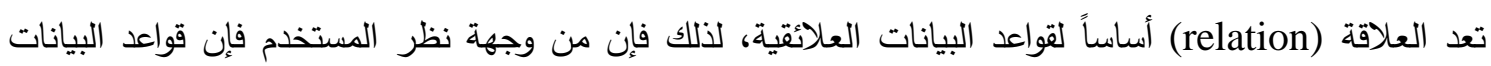

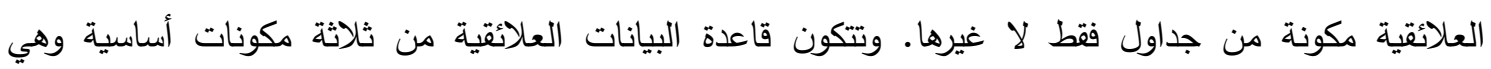
العلاقات (relations)، العمليات على تلك العلاقات (operation) وطرائق تكامل البيانات (data integrity) وبعبارٍ أخرى، توفر قواعد البيانات العلائقية مكاناً لحفظ واسترجاع البيانات وطريقةً لضمان تكامل البيانات. وتتميز

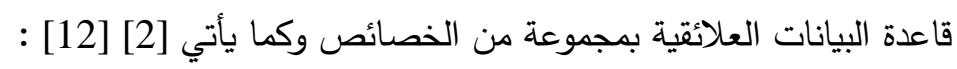
1- إن كل جدول يجب أن يكون له اسم فريد قاعدة البيانات.

2- العلاقة هي مصطلح رياضي وتمثل جدولاً ذا بعدين (صفوف وأعمدة).

3- ليس هناك ترتيب محدد للحقول وكذلك ليس هناك ترتيب محدد للسجلات وللأعمدة.

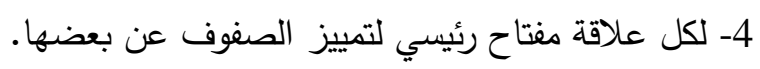

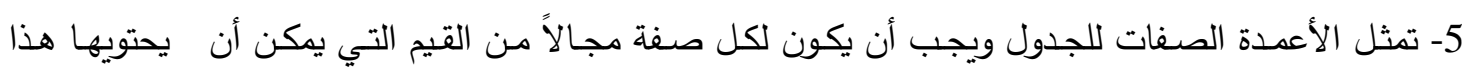

العمود.

6- درجة العلاقة هي عدد الأعمدة أو الصفات المكونة للجدول.

7- لكل حقل قيمة واحدة فقط.

10- تسمح قواعد البيانات العلائقية بمزج بيانات من جداول مختلفة للحصول على معلومات جديدة.

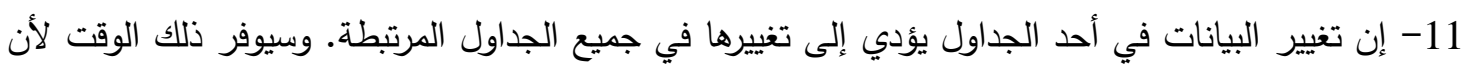
البيانات ستغير مرة واحدة فقط. والثكل (3) [2] يوضح أنموذجاً لجدول في قواعد البيانات العلائقية.

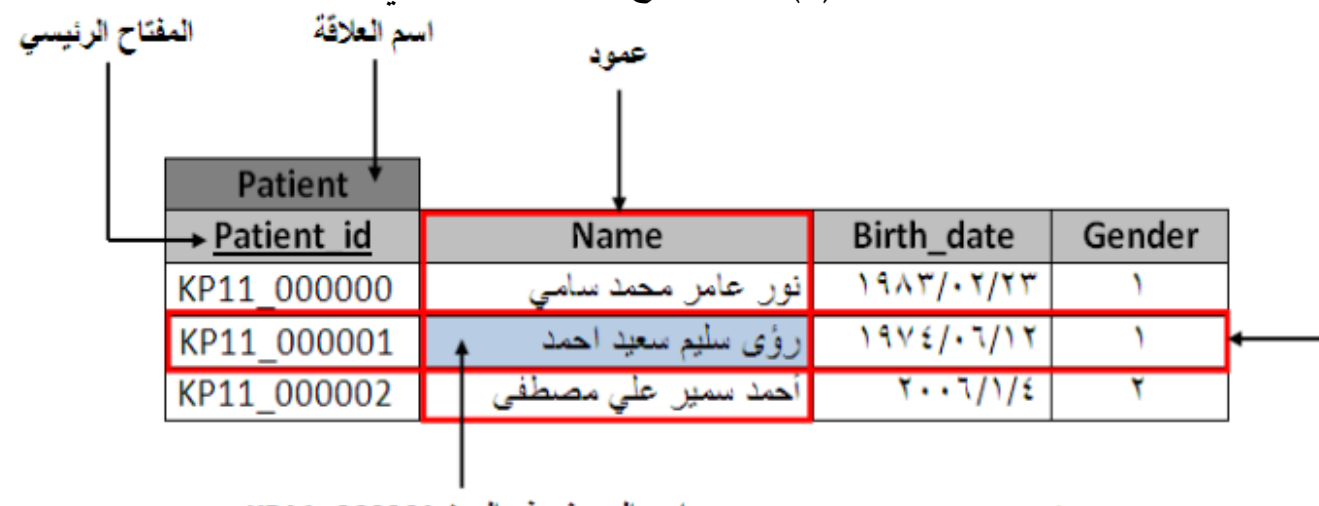

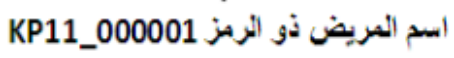

الثكل (3) أنموذج لجدول في قواعد البيانات العلائقية

Structured Query Language

SQL 5-5 لغة الإستفسار المهيكلة

هي لغة تستخدم لإصدار جميع الأوامر المتعلقة بقواعد، وتعود أصول هذه اللغة إلى نظام شركة IBM المسىى System R الذي تم تطويره في بداية الثمانينات بعد الاعتماد على الأنموذج النظري الذي قدمه الاكتور Edgar F. Codd التعديلات من قبل ANSI/ISO حتى استقرت تحت مسمى SQL، وتم اعتمداها لغة قياسية لأنظمة قواعد البيانات 
إن لغة SQL تعتمد على نظرية المجاميع (set theory) وتعد ميزة مهمة جداً حيث بإمكانها أن

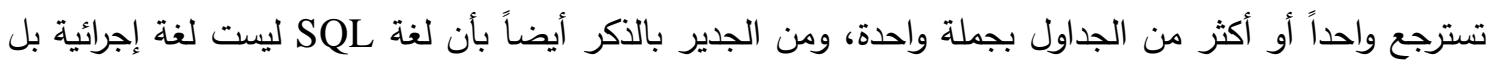

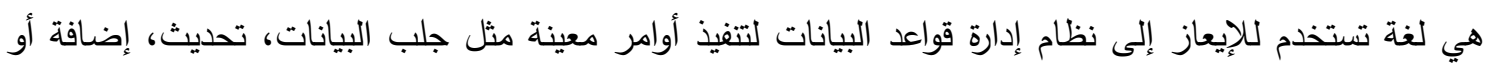

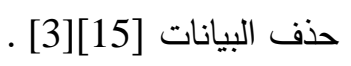

\section{Database Life Cycle}

2-5 دورة حياة قاعدة البيانات

من أجل بناء قاعدة البيانات بصورة مثالية يجب أن تمر بالعديد من المراحل الأساسية إبتداءً بدراسة

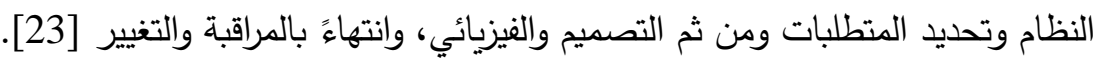

\section{Requirements Analysis}

5-2-1 تحليل المتطلبات

قبل البدء بتصديم أنظمة قواعد البيانات يجب دراستها ومعرفة الأهداف المرجوة منها والتعرف عن كثب على الاحتياجات المطلوبة لتصميمها وذلك بمقابلة الأشخاص الذين ينتجون البيانات التي سوف يغذى بها النظام وكذلك مقابلة المستخدمين للتعرف على متطلباتهم، تدوينها و دراستها جيداً. بعد ذلك يتم تكوين خطة التحليل

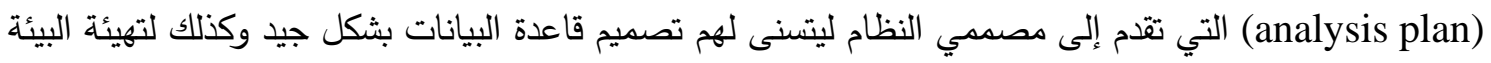

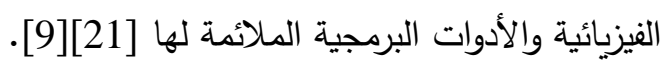

\section{Conceptual (Logical) Design}

5-2-5 التصميم المفاهيمي (المنطقي)

يهدف التصميم المفاهيمي إلى فصل قاعدة البيانات عن البرامج والتمثيل الفيزيائي لها ، وفيه تتم نمذجة جميع البيانات الخاصة بالنظام وتصميم أنموذج يمثل الكيانات والعلاقات التي تربطها بواسطة أدوات للتصميم مثل أنموذج الكيانات والعلاقات ERM ، بعدها يتم تحويل الأنموذج إلى جداول علائقية، وبشكل عام فإن التصميم المنطقي يوضح ماهية البيانات التي سوف يتم خزنها فعلياً في قاعدة ويهدف إلى البساطة والوضوح في فهم بنية

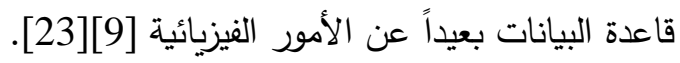

\section{Physical Design}

3-2-5

في هذه المرحلة يتم تحويل التصميم المنطقي لقاعدة البيانات إلى جداول باستخدام لغة تعريف البيانات الخاصة بنظام أدارة قاعدة البيانات، وبشكل عام فإن هذه المرحلة من التصميم تعنى بتحديد الطرائق

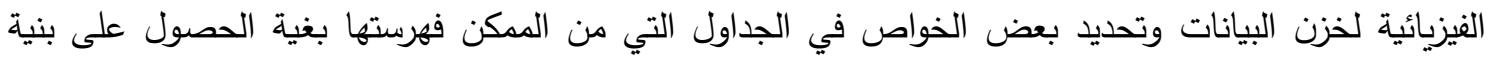

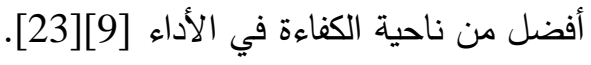

\section{Database implementation} 5-2-4 تمثيل قاعدة البيانات

إن تمثيل قاعدة البيانات هي مرحلة تطبيق النظام المصمم فيزيائياً على المؤسسة، وتعتمد هذه المرحلة

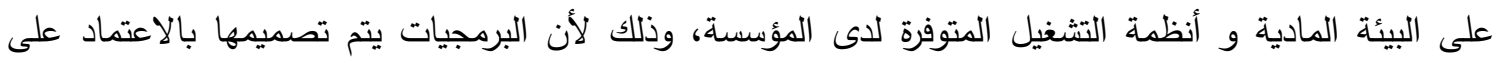

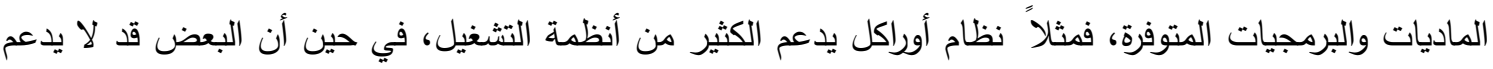
أكثر من نظام تشغيل واحد [9][23]. 
تبدأ عملية المراقبة عند قيام قاعدة البيانات بالعمل الفعلي في المؤسسة، وتقوم هذه المرحلة بإجراء

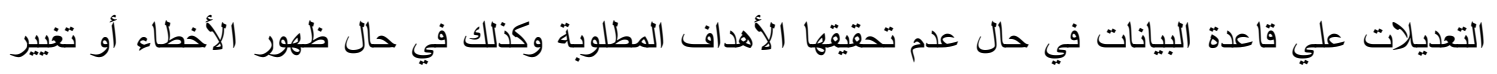

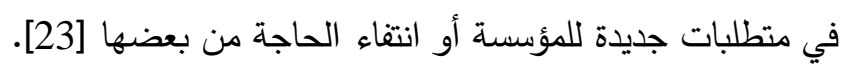

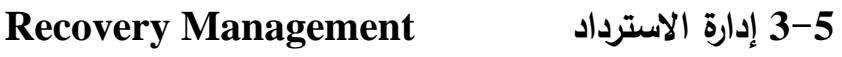

يعرف الاسترداد في قواعد البيانات بأنه إعادة قاعدة البيانات إلى آخر حالة صحيحة سابقة قبل وقت

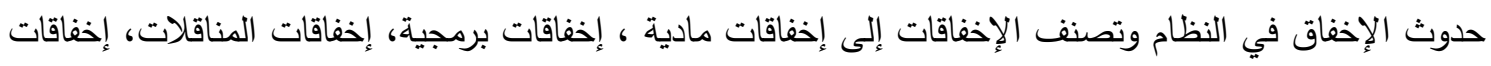

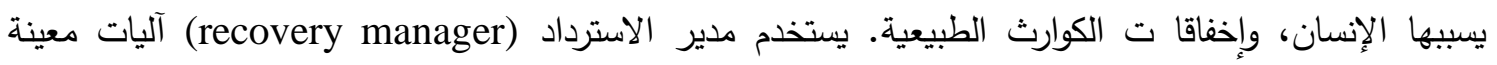

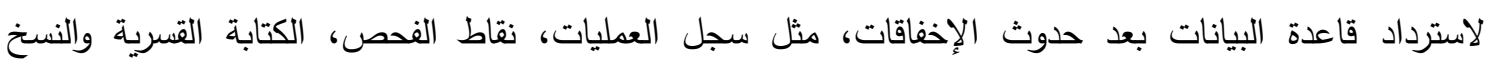

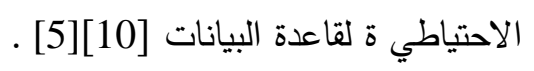

Distributed Databases 6- قواعد البيانات الموزعة

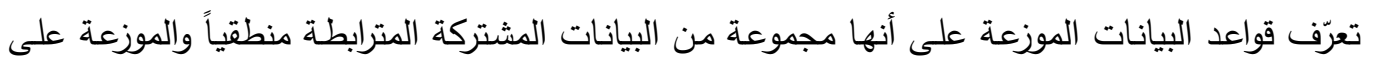
عدة مواقع (sites or nodes) في شبكة الحاسوب، ويتم إدارتها من قبل نظام إدارة قواعد البيانات الموزعة فئسة

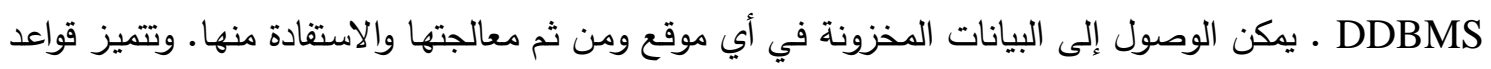

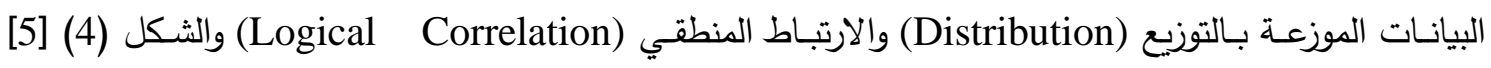
يوضح مفهوم قواعد البيانات الموزعة [5][10].

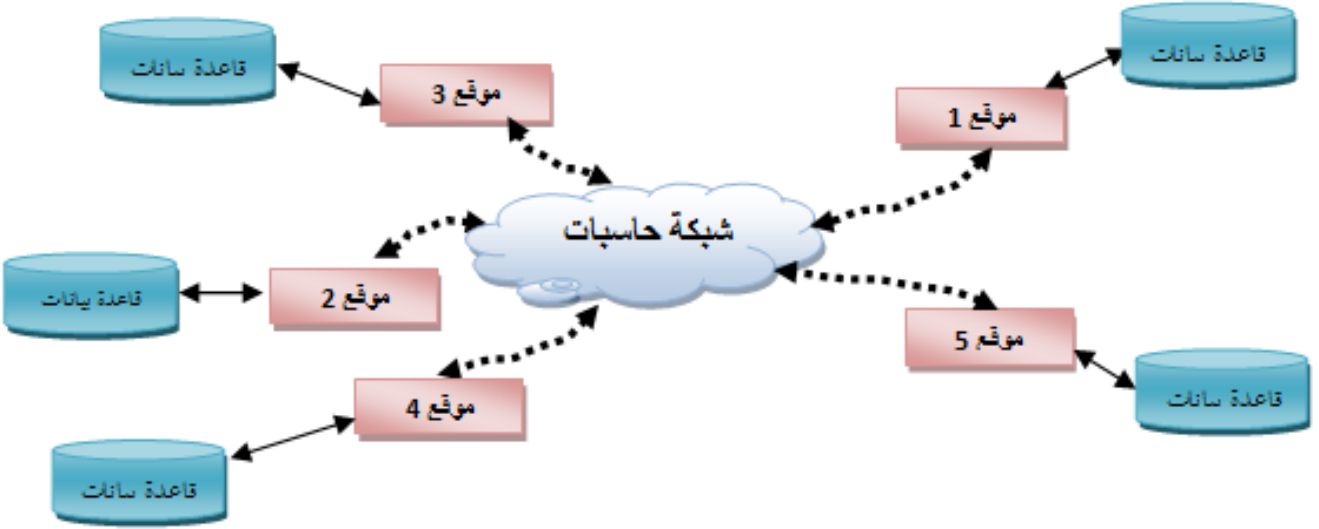

الثكل (4) قواعد البيانات الموزعة

\section{Classification of DDB}

6-6 تصنيف قواعد البيانات الموزعة

تصنف قواعد البيانات الموزعة إلى نوعين رئيسين اعتماداً على مدى تباين أنظمة إدارة قواعد البيانات في المواقع وكما يأتي [8] [6]: متماثلة Homogeneous: وفيها تكون قواعد البيانات متماثلة في كل موقع من ناحية نظام إدارة قواعد البيانات

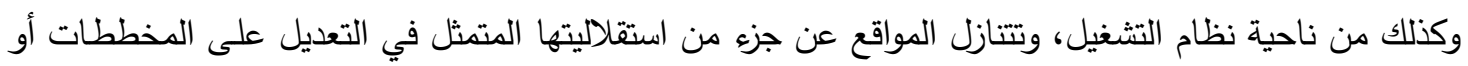

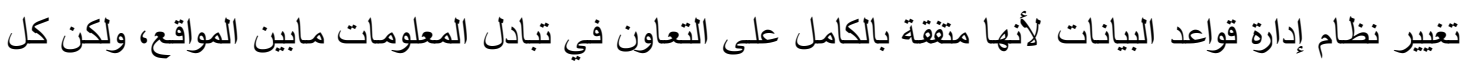
موقع يبقى مستقلاً في معالجة بياناته الخاصة. 
متغايرة Heterogeneous: قد تختلف المواقع في مخططاتها وفي نظم إدارة قواعد البيانات المستخدمة وحتى

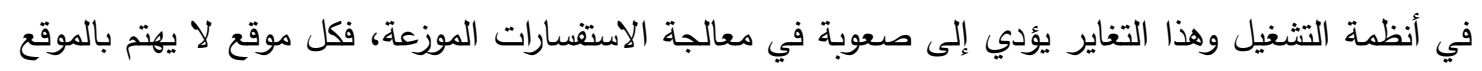
الآخر وقد يقدم تسهيلات بشكل محدود لتبادل المعلومات مع المواقع الأخرى.

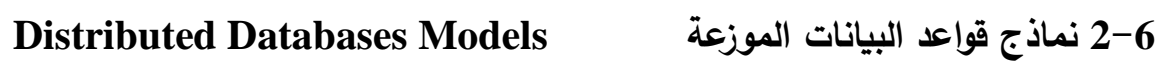

يوجد أنموذجان يمكن تطبيقهما في بناء أنظمة قواعد البيانات الموزعة وهما [4][1] :

Peer to Peer Model أنموذج النظير للنظير

وهو عبارة عن مجموعة من الحواسيب المتصلة مع بعضها البعض، ويختلف هذا الأنموذج عن النماذج

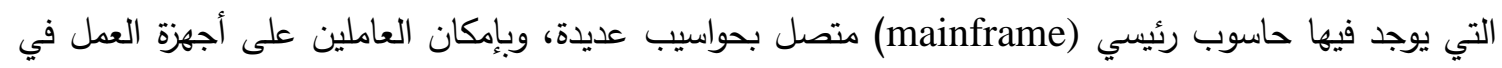

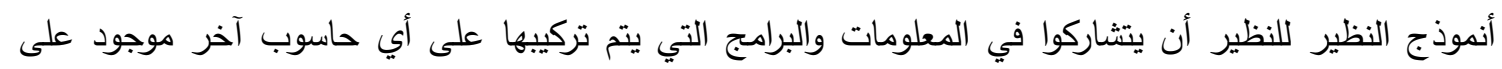

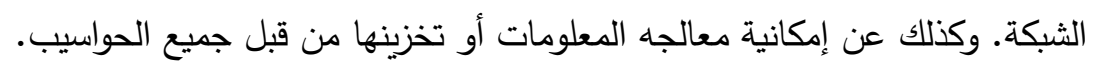

Client / Server Model أنموذج المستفيد / المزوّد

وهو الأنموذج المستخدم في الأعمال الحاسوبية الموزعة والأكثر تطوراً. إنّ المزود قد يكون جهاز حاسوب

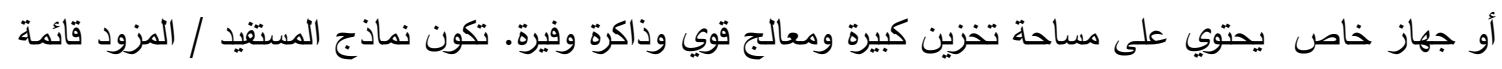

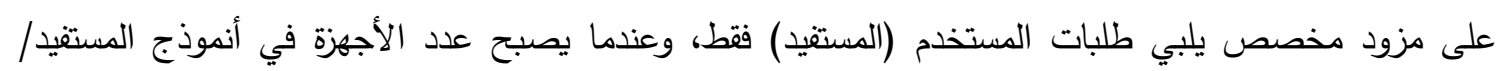

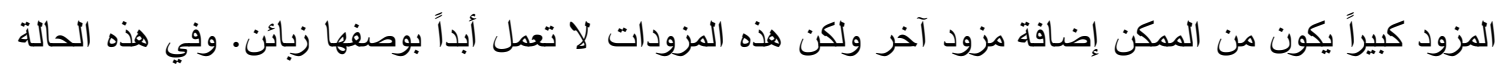

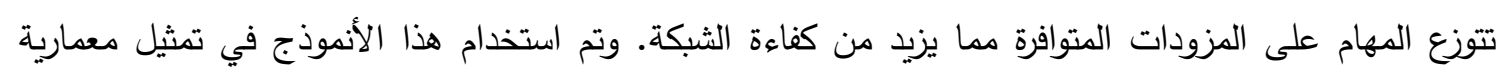

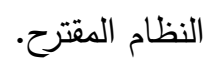

\section{Data Distribution Methods} 6-6 طرائق توزيع البيانات

تعد قاعدة البيانات موزعة عندما لا تكون البيانات متجمعة في موقع مركزي واحد وتكون الجداول موزعة

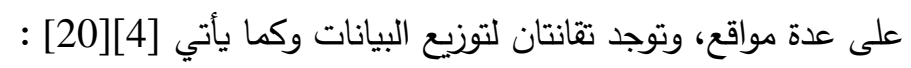

\section{Replication}

الاستنساخ

في بعض الأحيان، لا نحصل على نتائج مثلى للاستعلام، خصوصـا عندما تكون هناك قاعدة بيانات

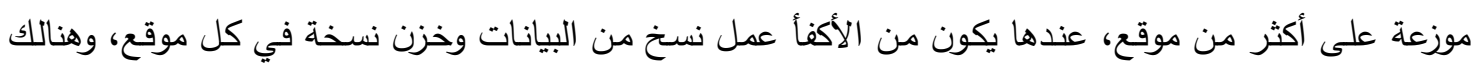

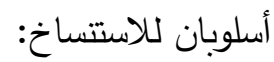
أ- الاستنساخ التام Full Replication: حيث يكون هنالك نسخة كاملة من قاعدة البيانات موجودة في عدة مواقع. وبإمكان هذا النوع من الاستنساخ أن يحسن مدى المتاحية على نحو ملحوظ لأنه يكون بإمكان النظام

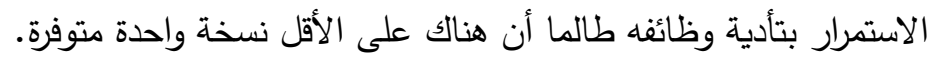
ب- الاستنساخ الجزئي Partial Replication: في هذا النوع من الاستنساخ بعض أجزاء فناء قاعدة البيانات تستتسخ والبعض الآخر لا يستسخ.

\section{Fragmentation التجزئة}


هي عملية تقسيم الجدول (العلاقة) إلى أجزاء أصغر وتوزيعها على المواقع المختلفة، وتكون التجزئة على التى ثلاثة أنواع هي التجزئة الأفقية (horizontal fragmentation)، التجزئة العمودية(

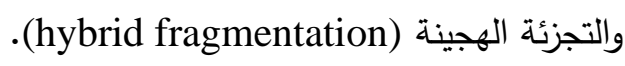

\section{Transparency}

4-6

من الأهداف المهمة في قواعد البيانات الموزعة إخفاء حقيقة بأن النظام الذي يعمل عليه مكون من

برمجيات وأجزاء مادية وبيانات موزعة على عدة مواقع، وتسمى هذه الخاصية بالثفافية وتتخذ عدة أشكال وكما يأتي

$:[6][22]$

• شفافية الوصسول Access Transparency: يقصد بها إخفاء الاختلافات في طريقة تمثيل و خزن

البيانات وطريقة الوصول إلى موارد النظام.

شفاف التكرار Replication Transparency:يجب أن يشعر المستخدم بأن البيانات واحدة ولا يوجد

لها نسخ أخرى.

• شفافية التجزئـة Fragmentation Transparency: يجب أن لا يعلم المستخدم بحقيقة تجزئـة

العلاقات أو الكيانات.

• شـفافية الموقـع Location Transparency: يجب أن لا يعلم المستخدم الموقـع الفيزيـائي لخزن

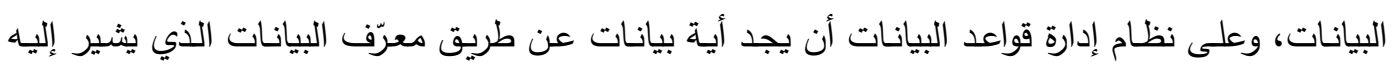

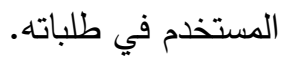

Implementation of The Proposed System

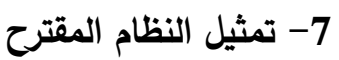

تمت زيارة وحدتي الأسنان في مستشفى الخنساء التعليمي في الموصل وفي مركز صحة المرأة بتاريخ

2010/10/12، وبعد مقابلة الأطباء والعاملين في وحدتي الأسنان، مشاهدة وتحليل آلية سير العمل، تحليل قوانين

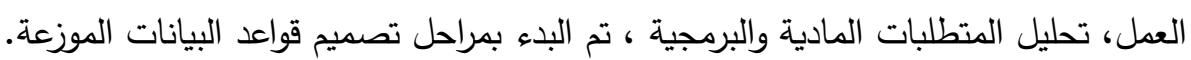

7-1 التصميم المنطقي Logical Design: بعد مشاهدة آلية العمل في وحدتي الأسنان منذ دخول المريض

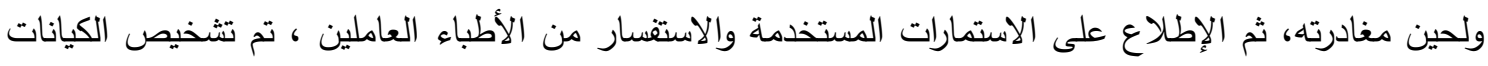
وهي (المرضى، الموظفون، المعالجات، الأمراض، الزيارات، الإجراءات، النشاطات الوقائية، النشاطات التثات التثقيفية) وكذلك تم تحديد صفاتها وكذلك العلاقات التي تربط تلك الكيانات مع بعض من خلال تتبع قوانين العمل.وتضمنت التهات تلك المرحلة تحويل الكيانات وصفاتها إلى علاقات (relations) وكما يأتي :

كيان المرضى Patients:

\begin{tabular}{|c|c|c|c|c|c|c|c|}
\hline Patients & \multicolumn{1}{|c|}{ Job } \\
\hline Patient ID & Chart_No & Name & Gender & BldType & Birth_Date & MarStat & Job \\
\hline PatPic & Address & Tel_No & EMail & RegDate & Organizer & & \\
\cline { 1 - 3 } & &
\end{tabular}

وبنفس الطريقة، تم تحويل الكيانات الأخرى (الموظفين، المعالجات، الأمراض، الزيارات، الإجراءات،

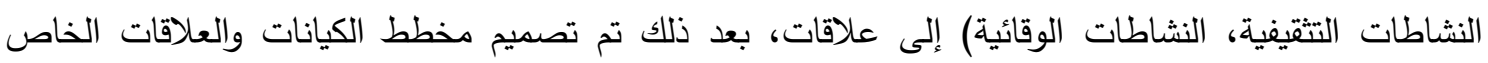

بالنظام وكما موضح في الثكل (5). 


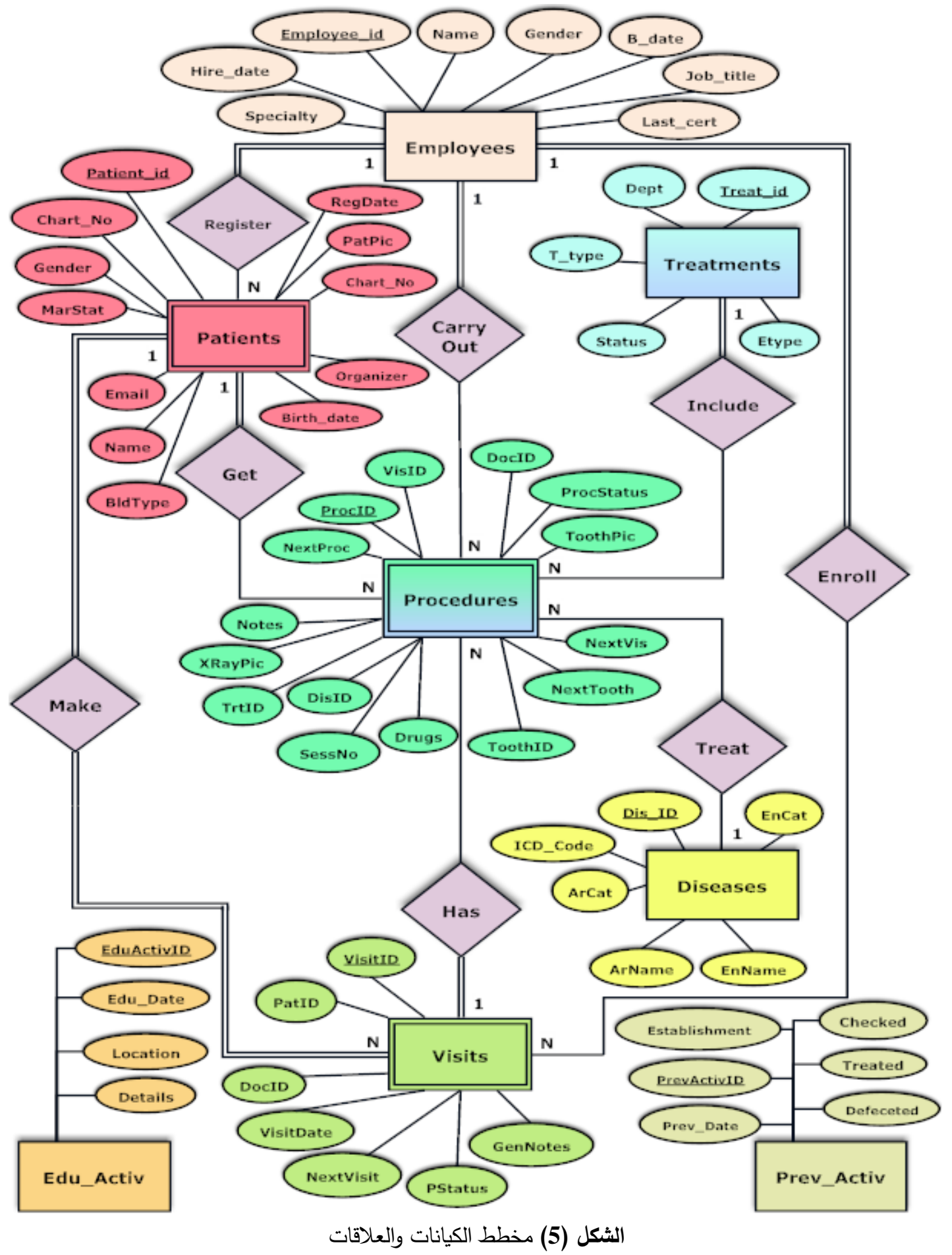

وبعد الإطلاع على النماذج المستحصلة من وحدتي الأسنان وآلية العمل، تبين أن الوحدتين متطابقتان

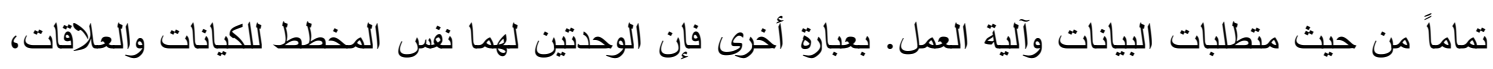
لاللك تم استخدام التجزئة الأفقية (horizontal distribution) لقاعدة البيانات لتكون على شكل جزأين موزعين على مزودي قاعدتي البيانات في وحدتي الأسنان. والثكل (6) يوضح معمارية النظام المقترح. 


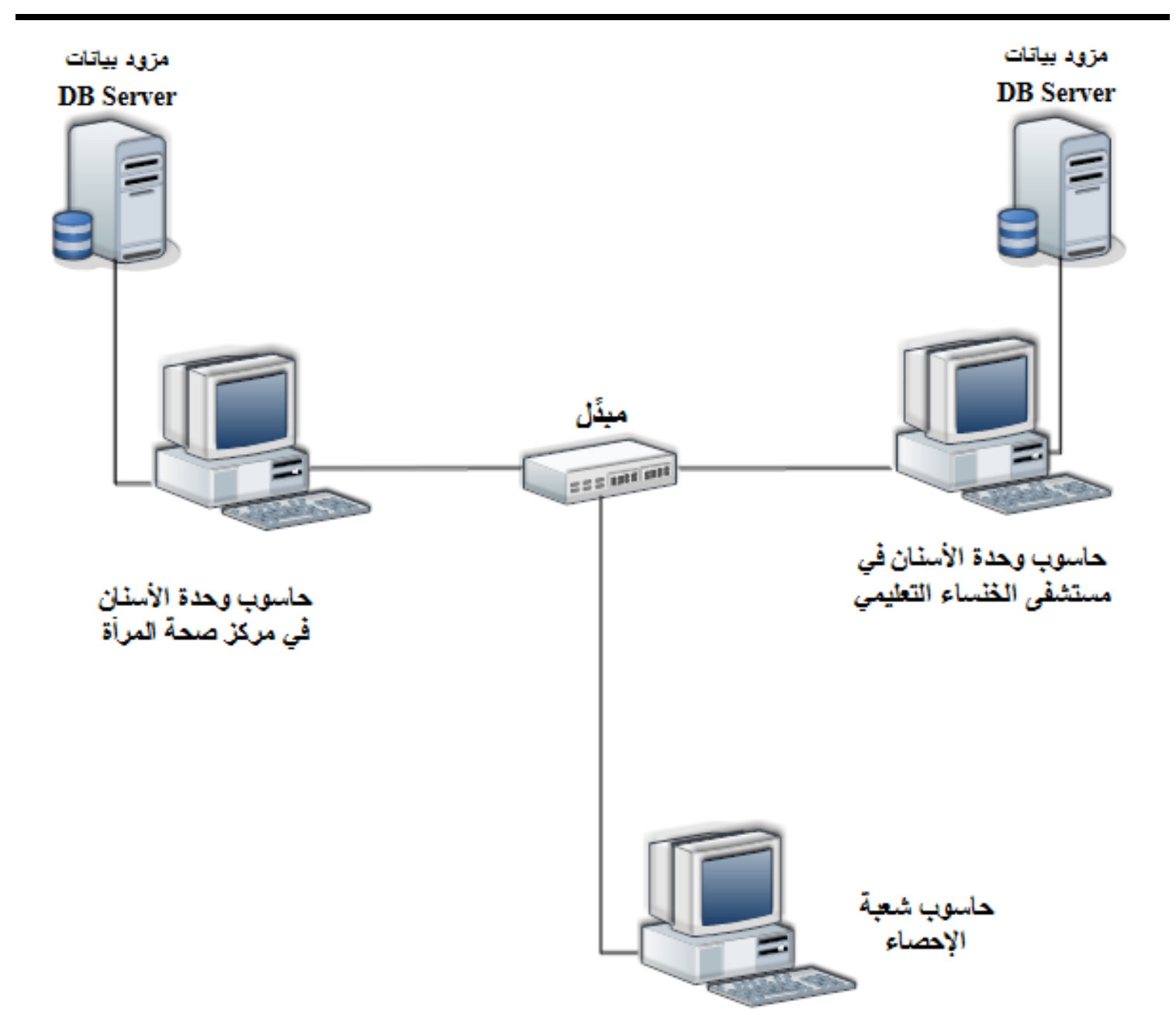

الثكل (6) معمارية النظام المقترح

\section{Physical Design of The Database}

7-7 التصميم الفيزيائي لقاعدة البيانات

شملت هذه المرحلة تحديد البنية الفيزيائية لقاعدة البيانات مثل الجداول وأنواع البيانات التي سوف تخزن في قاعدة البيانـات فضـلاُ عن تحديـد المشـاهد (views) وتطبيق قيود السـلامة وتكوين الفهارس (indexes)

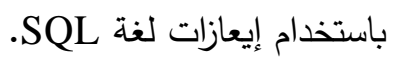

\section{Implementing The System}

3-7 تمثيل النظام

تم تجهيز البيئة المادية للنظام والتي تتكون من أجهزة الحاسوب وملحقاتها بالإضافة إلى تجهيز شبكة الحاسوب المحلية، فضلاً عن تجهيز البيئة والبرمجية للنظام والتي تتكون من نظام التثغيل Windows XP كبيئة لعمل النظام، واستخدمت برمجيات أوراكل (Oracle) في تمثيل النظام وبناء قواعد البيانات مثل قواعد بيانات أواركل بالإصدار 10g لبناء قواعد البيانات، فضلاً عن برمجيات أخرى من أوراكل مثل باني نماذجي فئن أوراكل لتصميم وبرمجة شاشات (واجهات) النظام المقترح، وباني تقارئ (Oracle Forms Builder)

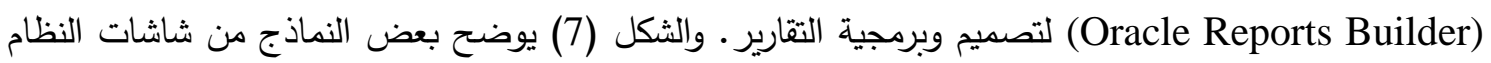



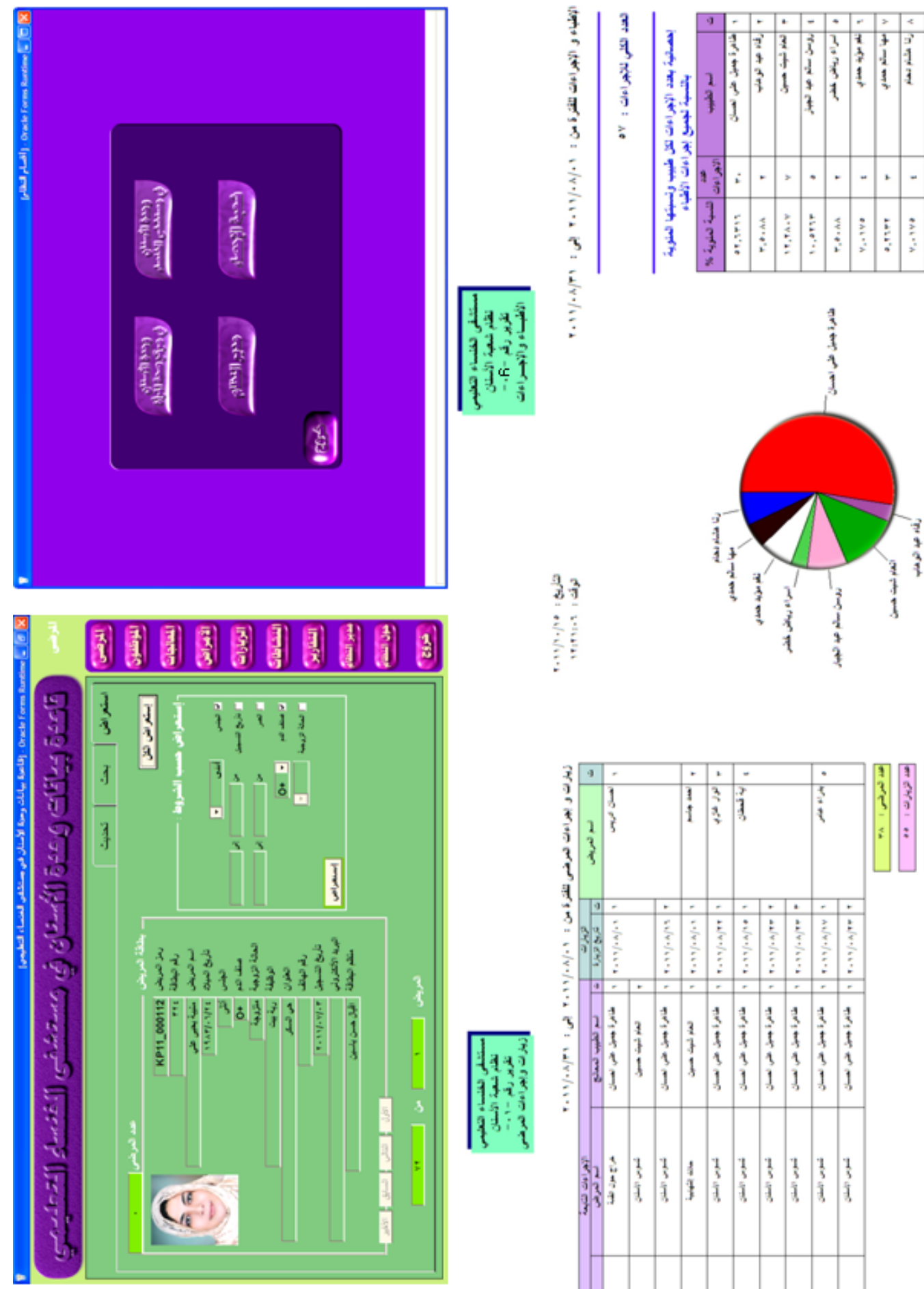

논

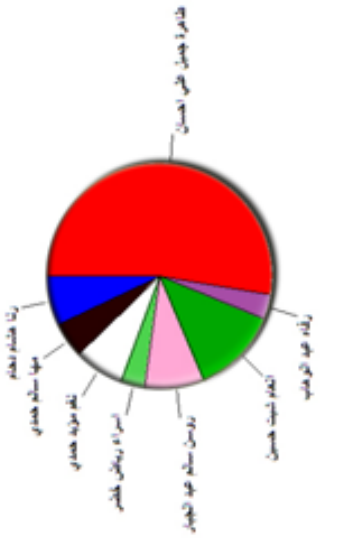

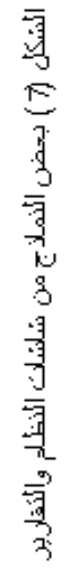

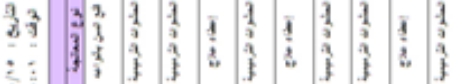




\section{Conclusions}

8- الاستنتاجات

بعد تصميم وتتفيذ نظام قواعد البيانات الموزعة واستخدامها في المجال الطبي تم إستتتاج الأمور الآتية: 1- إن عملية التحليل الصحيح للنظام القائم في المؤسسة، كان له أثر فاعل في تمثيل قواعد البيانات بصورة

$$
\text { صحيحة وفعالة. }
$$

2- ساعدت قواعد البيانات الموزعة على توزيع المهام على أكثر من موقع مما أدى إلى سرعة في إنجاز الأعمال

$$
\text { وتخفيف عبء المعالجة وتحقيق الخصوصية للبيانات. }
$$

3- يمتلك نظام أوراكل إمكانيات كبيرة في ربط قواعد البيانات الموزعة على الشبكة، كما يتمتع بأمنية عالية من

$$
\text { خلال نظام الصلاحيات التي يمتلكها. }
$$

4- حقق بناء هذا النظام أهدافه العامة المتمثلة في زج أنظمة المعلومات الموزعة ضمن المؤسسات الصحية

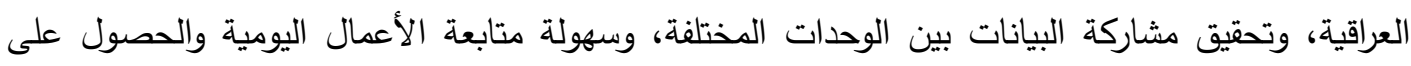
المعلومات، التقارير، واستخراج الإحصائيات الدورية بسهولة وسرعة مما ساعد على التوفير في الوقت والجهد.

5- حقق النظام الهدف الخاص منه وهو تصميم وتمثيل قواعد البيانات الموزعة بنجاح باستخدام أوراكل وبرمجياته الملحقة و تطبيق تقانات التوزيع بنجاح مثل تثانة التجزئة الأفقية وتطبيق الدفاهيم الأساسية لقواعد البيانات

$$
\text { الموزعة مثل الثفافية، النسخ الاحتياطي والاسترداد. }
$$

6- خلال العمل على تنفيذ النظام، تم ملاحظة بطء العاملين في المؤسسة في تقبلهم للعمل على الأنظمة

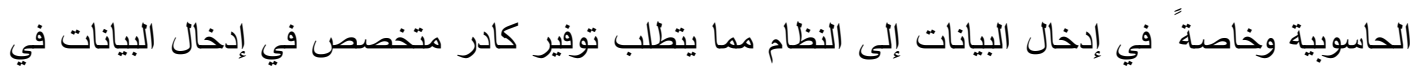
المؤسسات، وإدخالهم بدورات كفاءة قبل بدئهم العمل على النظام.

7- إن وضع فترة تجريبية لعمل النظام ساعد في حصر الأخطاء وإضافة المتطلبات الجديدة للمستفيدين والمستخدمين والتي تبرز أثناء العمل والقيام بتصحيح مسار النظام لتقبل الآراء المختلفة من المستفيدين لكي يصبح النظام الحاسوبي مقبولاً للعمل عليه. 


$$
\begin{aligned}
& \text { المصادر } \\
& \text { الليلة، ظفر فخري حسن، "تصميم وتطبيق قواعد البيانات الموزعة". رسالة ماجستير ، كلية علوم الحاسبات } \\
& \text { والرياضيات، جامعة الموصل (2002). } \\
& \text { المؤسسة العامة للتعليم الفني والتدريب المهني، "تصميم قواعد البيانات". المؤسسة العامة للتعليم الفني } \\
& \text { والتدريب المهني، المملكة العربية السعودية (2005). } \\
& \text { المؤسسة العامة للتعليم الفني والتدريب المهني، "مقدمة قواعد بيانات أوراكل". المؤسسة العامة للتعليم } \\
& \text { الفني والتدريب المهني، المملكة العربية السعودية (2005). } \\
& \text { رزوقي، شيماء أحمد، "استخدام قواعد بيانات موزعة لتطوير نظام لإدارة كلية علوم الحاسبات } \\
& \text { والرياضيات". رسالة ماجستير، كلية علوم الحاسبات والرياضيات، جامعة الموصل (2009). } \\
& \text { سعيد، أيوب علي محمد، "تصميم وتطبيق نظام موزع وقاعدة بيانات موزعة لأعمال دائرة المرور". رسالة } \\
& \text { ماجستير ، كلية علوم الحاسبات والرياضيات، جامعة الموصل (2010). } \\
& \text { ياسين، عمار ظاهر ، "تصميم وتتفيذ قواعد بيانات موزعة لأعمال البنوك باستخدام الزمن الحقيقي". } \\
& \text { رسالة ماجستير، كلية علوم الحاسبات والرياضيات، جامعة الموصل (2009). }
\end{aligned}
$$

[7] Alkhatib, G., Labban, R. S.,2002 "Transaction Management in Distributed Database Systems: the Case of Oracle's Two-Phase Commit" Journal of Information Systems Education, Vol. 13, No 2, Pages 95-102, January 1, (2002).

[8] Beasley, J. S., "Networking". 2nd Edition, Pearson Education Inc (2009).

[9] Buxton, S., Nadeau, T. P., Fryman, L., O'Neil, B., Güting, R. H., O'Neil, E.,Halpin, T., O'Neil, P., Harrington, J. L., Schnieder, M., Inmon, W. H., Simsion, G., Lightstone, S. S., Teorey, T. J., Melton, J., Witt, G., Morgan, T., "Database Design Know it All". Morgan Kaufmann Publishers (2009).

[10] Elmasri, R., Navathe, S. B., "Fundamentals of Database Systems". 6th Edition, Addison Wesley (2010).

[11] Forouzan, B. A., "Data Communications and Networking". 4th Edition, McGraw-Hill (2007).

[12] Harrington, J. L., " Relational Database Designing and Implementation". 3rd Edition, Morgan Kaufmann (2009).

[13] Held, G., "Ethernet Networks". Fourth Edition, John Wiley \& Sons, Ltd (2003).

[14] Khair, M., Mavridis, I., Pangalos, G., "Design of Secure Distributed Medical Database Systems". Lecture Notes in Computer Science, Database and Expert Systems Applications, 9th International Conference, DEXA'98, Vienna, Austria, Pages 492- 500 (1998).

[15] Loney, K. , "Oracle Database 10g: The Complete Reference". McGraw- Hill (2004). 
[16] Malinowski E., Chakarvarthy S., "Fragmentation Techniques". Springer-Verlag London UK , Proceeding ER '97 Proceedings of the 16th International Conference on Conceptual Modeling, Pages 347 - 360 (1997) .

[17] Özsu, M. T., Valduriez, P., "Priciples of Distributed Database Systems". Third Edition, Springer Science+Business Media LLC (2011).

[18] Rababaah, H., "Distributed Databases Fundamentals and Research". Department of computer and information sciences, Indiana University South Bend (2005).

[19] Rahimi, S. K., Haug, F. S. ,"Distributed Database management Systems". Wiley \& Sons, Inc (2010).

[20] Singh, R., Singh, G., Pannu, V., "Optimized Access Strategies for a Distributed Database Design". International Journal of Data Engineering (IJDE), Vol. 2 : Issue (3), Pages 102-110 (2011).

[21] Stephens, R. K., Plew, R. R., "Database Design". Sams Publishing (2001).

[22] Tanenbaum, A. S., Steen, M. V., "Distributed systems : Principles and Paradigms". Prentice Hall (2002).

[23] Teorey, T., Lightstone, S., Nadeau, T., Jagadish, H. V., "Database Modeling And Design". 5th Edition, Elsvier Inc (2011).

[24] Taylor A. G., "SQL for Dummies". Seventh Edition, John Wiley \& Sons Inc. (2010). 